\title{
Global numerical simulations of differentially rotating disks with free eccentricity
}

\author{
J. C. B. Papaloizou
}

\author{
Astronomy Unit, Queen Mary, University of London, Mile End Rd, London E1 4NS, UK \\ e-mail: jcbp@maths.qmw.ac.uk
}

Received 3 September 2004 / Accepted 10 December 2004

\begin{abstract}
We study the nonlinear evolution of global $m=1$ modes with low pattern speeds in a differentially rotating non magnetic disk, by means of two and three dimensional numerical simulations. The modes make disk streamlines eccentric with maximum eccentricity in the range $0.13-0.24$.

We found that long lived patterns corresponding to eccentricities $\sim 0.1$ lasted for the duration of the simulations of $\sim 64$ orbits at the disk outer boundary. They had slow retrograde precession with period $\sim 10$ orbits at the outer boundary, in good agreement with that found from linear normal mode analysis.

As expected from linear stability analysis, which leads one to expect a parametric instability associated with the non circular streamlines, we also found that three dimensional simulations showed the growth of a local instability to producing vertical motions on a local scale that eventually results in small amplitude turbulence with root mean square vertical velocity typically $\sim 0.03 c_{\mathrm{s}}, c_{\mathrm{s}}$ being the sound speed. This turbulence together with much more effective shocks produced by the interaction of the eccentric disk with the inner boundary were responsible for damping the disk eccentricity. Our first estimate of the damping time associated with turbulence for eccentricities $\sim 0.1$, corresponded to that associated with a turbulent viscosity acting to circularize the eccentric streamlines with " $\alpha$ " parameter $\sim 10^{-3}$. For parameters appropriate to protostellar disks at 5 AU this corresponds to a decay time $\sim 10^{5-6} \mathrm{y}$.

Thus although the indication is that disks with eccentric streamlines are long lived, there is associated turbulence even in the non magnetic case leading to estimated decay times that may be significant when the possibility of the growth of orbital eccentricity for extrasolar planets is considered through disk planet interaction on sufficiently long timescales.
\end{abstract}

Key words. accretion, accretion disks - instabilities - hydrodynamics - celestial mechanics - planets and satellites: formation

\section{Introduction}

Astrophysical systems such as stars undergoing pulsation or accretion disks with excited global modes which make their streamlines non circular are susceptible to parametric instabilities (see a preceding paper, Papaloizou 2005, hereafter Paper I and references therein). In Paper I, we formulated a general local linear stability analysis which regarded the perturbations as being localized in the neighbourhood of fluid streamlines. We also delineated the generic parametric instability in the case of a simple example for which the streamlines were Keplerian ellipses with eccentricity, $e$, up to 0.7 .

The instability is local and thus may be confined to the neighbourhood of streamlines that do not suffer instability from other causes such as the presence of a magnetic field. It could thus be of potential interest in the regions of protostellar disks that do not support magnetic fields in situations where the streamlines are non circular.

Disks with eccentric streamlines may arise in a number of astrophysical contexts (see e.g. Ogilvie 2001; and Paper I for more discussion). In particular the discovery of extrasolar giant planets orbiting around nearby solar-type stars
(Mayor \& Queloz 1995; Marcy \& Butler 1998, 2000) with high eccentricities $>0.2$ has led to the suggestion that disk planet interaction could be responsible for their origin. This leads one to consider the possibility of excitation of normal modes involving eccentricity of the disk and planet orbit together (e.g. Goldreich \& Sari 2003; and see Paper I for more discussion). Hence the behaviour of free disk global normal modes is of interest in this context.

It is the purpose of this paper to study such modes and to investigate the non linear outcome of any local instabilities by means of two and three dimensional hydrodynamic simulations. The two dimensional simulations of course do not show parametric instability and so provide a reference point for the three dimensional simulations which do.

We proceed by studying the nonlinear evolution of global $m=1$ modes with low pattern speeds in differentially rotating disks. These modes correspond to distorting the streamlines from circles into ellipses. In the absence of pressure the pattern produced would be stationary in the inertial frame. In addition ellipses at different radial locations would be independent. However, the introduction of pressure enables a coherent 
normal mode and introduces in general, a very slow retrograde pattern rotation.

In order to connect with linear oscillation theory for a disk model, we calculate a linear normal mode independently and use that to construct initial conditions for the non linear multidimensional simulations. In this way the ability of the simulation to obtain the correct linear behaviour, including the correct pattern speed for the mode, can be verified. The modes considered here involve radial motions appropriate to maximum disk eccentricities in the range $0.13-0.24$.

In the examples with larger eccentricity, shock dissipation was effective at producing decay. However, modes corresponding to eccentricities $\sim 0.1$ survived for $\sim 64$ orbits at the disk outer boundary being the simulation run time.

Three dimensional simulations, that allow vertical motion, show the growth of a local instability that leads to turbulence with root mean square vertical velocity typically $\sim 0.03 c_{\mathrm{s}}, c_{\mathrm{s}}$ being the sound speed. In the simulations performed here, the turbulence was apparently much less effective at damping the eccentricity than shocks induced by the presence of the inner boundary. Nonetheless the damping time estimated to be associated with turbulence for a disk with $e \sim 0.1$, under conditions appropriate for protostellar disks at $5 \mathrm{AU}$ was estimated to be $\sim 10^{5-6} \mathrm{y}$. Thus the existence of this type of turbulence may need to be taken into account when considering evolutionary phenomena in eccentric disks including the eccentricity excitation itself (e.g. Artymowicz 1992; Goldreich \& Sari 2003) should this occur on sufficiently long timescales.

The plan of this paper is as follows. In Sect. 2 we give the basic equations In Sect. 3 we describe the linear analysis applicable to modes with $m=1$ which have very small pattern speed and the imposition of which corresponds to giving the fluid trajectories a small eccentricity. In Sect. 4 we describe the nonlinear numerical simulations discussing their relationship to the linear normal modes and the onset and effects of parametric instability. Finally in Sect. 5 we summarize our results giving a discussion in the context of protostellar disks and extrasolar planets.

\section{Basic equations}

We work in a non rotating cylindrical coordinate system $(r, \varphi, z)$ with origin at the primary star approximated as a point mass. The components of the basic equations of motion can be written in the form

$$
\begin{aligned}
& \frac{\partial v_{r}}{\partial t}+v_{r} \frac{\partial v_{r}}{\partial r}+\frac{v_{\varphi}}{r} \frac{\partial v_{r}}{\partial \varphi}+v_{z} \frac{\partial v_{r}}{\partial z}-\frac{v_{\varphi}^{2}}{r}=-\frac{1}{\rho} \frac{\partial P}{\partial r}-\frac{\partial \Psi}{\partial r} \\
& \frac{\partial v_{\varphi}}{\partial t}+v_{r} \frac{\partial v_{\varphi}}{\partial r}+\frac{v_{\varphi}}{r} \frac{\partial v_{\varphi}}{\partial \varphi}+v_{z} \frac{\partial v_{\varphi}}{\partial z}+\frac{v_{\varphi} v_{r}}{r}=-\frac{1}{r \rho} \frac{\partial P}{\partial \varphi}-\frac{1}{r} \frac{\partial \Psi}{\partial \varphi} \\
& \frac{\partial v_{z}}{\partial t}+v_{r} \frac{\partial v_{z}}{\partial r}+\frac{v_{\varphi}}{r} \frac{\partial v_{z}}{\partial \varphi}+v_{z} \frac{\partial v_{z}}{\partial z}=-\frac{1}{\rho} \frac{\partial P}{\partial z}-\frac{\partial \Psi}{\partial z}
\end{aligned}
$$

In addition we have the continuity equation

$$
\frac{\partial(r \rho)}{\partial t}+\frac{\partial\left(r \rho v_{r}\right)}{\partial r}+\frac{\partial\left(\rho v_{\varphi}\right)}{\partial \varphi}+\frac{\partial\left(r \rho v_{z}\right)}{\partial z}=0
$$

Here the velocity is $\boldsymbol{v}=\left(v_{r}, v_{\varphi}, v_{z}\right)$. The density is $\rho$ and the pressure is $P$. For the work presented here we adopt a locally isothermal equation of state such that

$P=\rho c_{\mathrm{s}}^{2}(r)$.

The sound speed $c_{\mathrm{S}}(r)$ is taken to be a fixed function of $r$. The gravitational potential is $\Psi$. We neglect the self-gravity of the disk so that $\Psi$ is produced entirely by the central star. In order to make the computational requirements tractable we use cylindrical disk models for which the stratification is neglected (e.g. Hawley 2000; Steinacker \& Papaloizou 2002). The potential is then independent of $z$ such that $\Psi=-G M_{*} / r$, with $M_{*}$ being the central mass and $G$ the gravitational constant. In addition, for the particular models we later consider numerically, we adopted $c_{\mathrm{s}}^{2}=0.0144 G M_{*} / r$ corresponding to a putative uniform aspect ratio $H=c_{\mathrm{s}} /(r \Omega) \equiv h=0.12$.

The equation of state (5) or its two dimensional equivalent have been commonly used in simulations of disk planet interactions (e.g. Kley 1999; Bryden et al. 1999; Nelson et al. 2000; Masset 2001; Papaloizou \& Nelson 2003; Nelson \& Papaloizou 2003; Winters et al. 2003). It results in no effective stratification in either the radial or vertical direction, independently of the cylindrical disk prescription. Thus there can be no convective instability. In this respect the fluid behaves as one with zero entropy gradient (also see Paper I).

For all models considered here, the density is initially given by the linear form

$\rho=\rho_{0} \frac{r}{R_{\text {in }}}$,

where $R_{\text {in }}$ is the inner boundary radius and $\rho_{0}$ is a constant. As we neglect the disk self-gravity this constant is arbitrary and can be used to scale the total disk mass. In this set up the pressure, $P$, is constant and the disk angular velocity is Keplerian with $\Omega=\sqrt{G M_{*} / r^{3}}$.

We chose a density that increases with radius because this may be a reasonable assumption for conditions just exterior to a planet that has induced a cavity interior to the disk and which participates in the normal mode responsible for the free eccentricity of the disk (Papaloizou 2002).

Although a cylindrical disk model may not be as realistic as a vertically stratified model (see e.g. Ogilvie 2001), there is significant reduction in computational overhead enabling a wider study. Furthermore the parametric instability we study is local and representable in a cylindrical disk model where conditions resemble those in the midplane of a vertically stratified model. Useful advantages are that a linear global normal mode is easily calculated for comparison with the simulations and we can compare three dimensional models with turbulence with equivalent two dimensional models without and in the process get a measure of the effects of the turbulence.

\section{Linear analysis}

Here we are interested in disks with a global eccentricity. In terms of linear normal modes such a disturbance corresponds to a mode with azimuthal mode number $m=1$ (see e.g. 
Papaloizou 2002). When this corresponds to motion in the disk close to that of particles on Keplerian elliptical orbits, the mode has a slowly varying pattern when viewed in an inertial frame. The elliptical pattern thus precesses on a timescale much longer than the orbital period.

\subsection{Slowly varying modes with $m=1$}

To describe such normal modes, we linearize the basic equations about the above cylindrical disk equilibrium for which $\rho=\rho(r), P=P(r)$ and $v_{\varphi}=v_{\varphi}(r) \equiv r \Omega$, with other velocity components being zero. We adopt the locally isothermal equation of state (5) throughout.

We denote perturbations to quantities with a prime making the usual assumption that the dependence of all perturbations on $\varphi$ and $t$ is through a factor $\exp i\left(m\left(\varphi-\Omega_{P} t\right)\right)$. Due to the symmetry of cylindrical disk models we are able to consider perturbations which do not depend on $z$. We further specialize to the case $m=1$ and slowly varying pattern speed $\Omega_{P} \ll$ $\Omega$. Here we shall ignore the possible participation of planets orbiting in an inner cavity (Papaloizou 2002).

The linearized forms of Eqs. (1), (2) and (4) are then

$\operatorname{im}\left(\Omega-\Omega_{P}\right) v_{r}^{\prime}-2 \Omega v_{\varphi}^{\prime}=-c_{\mathrm{s}}^{2} \frac{\partial W}{\partial r}$,

$\operatorname{im}\left(\Omega-\Omega_{P}\right) v_{\varphi}^{\prime}+\frac{\kappa^{2}}{2 \Omega} v_{r}^{\prime}=-\frac{\operatorname{im} c_{\mathrm{s}}^{2} W}{r}$,

and

$\operatorname{im}\left(\Omega-\Omega_{P}\right) \rho W=-\frac{1}{r} \frac{\partial\left(r \rho v_{r}^{\prime}\right)}{\partial r}-\frac{\operatorname{im} \rho v_{\varphi}^{\prime}}{r}$.

Here $W=\rho^{\prime} / \rho$, and $\kappa^{2}=(2 \Omega / r)\left(d\left(r^{2} \Omega\right) / d r\right)$ denotes the square of the epicyclic frequency.

\subsection{Slowly varying modes with $m=1$}

We here focus on modes such that $\Omega_{P} \ll \Omega$. Then, assuming highly supersonic Keplerian rotation, to lowest order we may neglect $c_{\mathrm{s}}^{2}$ and $\Omega_{P}$ to obtain from Eq. (8)

$i v_{\varphi}^{\prime}=-\frac{1}{2} v_{r}^{\prime}$.

Using this Eq. (4) gives the density perturbation in the low frequency limit in the form

$\rho^{\prime}=\frac{i}{r^{1 / 2} \Omega} \frac{\mathrm{d}\left(\rho r^{1 / 2} v_{r}^{\prime}\right)}{\mathrm{d} r}$.

Eliminating $v_{\varphi}^{\prime}$ from Eqs. (7) and (8), using Eq. (11) for $\rho^{\prime}$ and expanding to first order in the small frequency $\Omega_{P}$, one obtains a normal mode equation for $v_{r}^{\prime}$ in the form of a second order differential equation (see also Papaloizou 2002; Papaloizou et al. 2001)

$\frac{\mathrm{d}}{\mathrm{d} r}\left(r^{2} \frac{\mathrm{d}\left(v_{r}^{\prime} r^{3 / 2}\right)}{\mathrm{d} r}\right)=\frac{2 \Omega_{P} v_{r}^{\prime} r^{3 / 2}}{\Omega h^{2}}$.

When we are concerned with simple boundary conditions on $v_{r}^{\prime}$ such as those adopted here, that it be zero at the ends of the finite domain considered, we may regard $\Omega_{P}$ as an eigenvalue associated with a standard Sturm-Liouville problem.

\subsection{Normal modes}

We have calculated the lowest order normal modes, for the disk models we consider, by solving the eigenvalue problem specified by the second order ordinary differential Eq. (12) together with the above boundary conditions numerically.

We obtain for the fundamental mode (with no nodes in $r$ ) appropriate to all models other than C3 that $2 \Omega_{P} / h^{2}=$ $-1.917 \sqrt{G M_{*} / R_{\text {in }}^{3}}$. The negative sign corresponds to a retrograde pattern speed with a period of one rotation given by $P_{P}=2 \pi /\left|\Omega_{P}\right|=6.555 h^{-2} \sqrt{R_{\text {in }}^{3} / G M_{*}}$. For model C3 which has a more extended outer boundary, we find the corresponding period to be $P_{P}=2 \pi /\left|\Omega_{P}\right|=10.097 h^{-2} \sqrt{R_{\text {in }}^{3} / G M_{*}}$. For all models other than $\mathrm{C} 3$, the period of the first overtone with one node in $r$ was found to be given by $P_{P}=2 \pi /\left|\Omega_{P}\right|=$ $1.6592 h^{-2} \sqrt{R_{\text {in }}^{3} / G M_{*}}$. This was also retrograde.

Some of the properties of these modes even though they are global, can be understood with reference to the local dispersion relation for density waves in the low frequency limit without self-gravity (e.g. Lin \& Shu 1969; Papaloizou 2002) that can be obtained from equation (13) after substituting $v_{r}^{\prime} \propto \exp (i k r)$ and assuming that the radial wavenumber, $k$ is very large, in the form

$2 \Omega_{P}=-\Omega h^{2}(k r)^{2}$.

This indicates that $\Omega_{P}$ is negative. implying that the direction of pattern rotation should be retrograde as is the case.

\section{Nonlinear numerical simulations}

The numerical method is that outlined in Hawley \& Stone (1995) and implemented in the ZEUS code. The code has been developed from a version of NIRVANA originally written by U. Ziegler (see Ziegler \& Rüdiger 2000, and references therein). It has been used in a number of three dimensional disk simulations, with and without internal planets and with and without MHD. (Kley 1999; Steinacker \& Papaloizou 2002; Nelson \& Papaloizou 2004; Papaloizou et al. 2005). In this first study of the behaviour of differentially rotating disks with free eccentricity, we neglect MHD as may be reasonable in the regions of protostellar disks with low ionization, leaving the effect of the incorporation of magnetic fields to a future study.

\subsection{Initial disk models}

We present here results obtained for disk models with the locally isothermal equation of state given by Eq. (5).

The radius of the outer boundary of the computational domain is $R_{\text {out }}$. This is such that $R_{\text {out }} / R_{\text {in }}=3.8$ for all models other than C3 (see below). In that case $R_{\text {out }} / R_{\text {in }}=4.6$. The radial boundaries at $r=R_{\text {in }}$ and $r=R_{\text {out }}$ were taken to be rigid. Experiments with open boundaries indicated that reasonable models of eccentric disks, with the rather high pressures adopted here, could not be sustained for adequate time periods due to serious losses of mass and kinetic energy through such boundaries. For example in the case of model C (see below), 
the radial kinetic energy was halved after 12 orbits at the inner boundary and the total mass was halved after about 30 such orbits. Accordingly open boundaries were not used. The vertical domain is taken to be $\left(z_{1}, z_{2}\right)$ with $z_{2}=-z_{1}=H_{1}=0.12 R_{\text {in }}$. Periodic boundary conditions are applied at the vertical boundaries. The azimuthal domain is $(0,2 \pi)$. A uniform computational mesh is used with 350,400, and 35 grid points in the $r$, $\varphi$, and $z$ directions respectively for all models other than $\mathrm{C} 3$ which differed by having 450 grid points in the radial direction on account of the larger radial domain in that case. With these meshes the spacing in the radial and vertical directions are approximately the same with 30 and 35 grid points spanning the vertical domain $z_{2}-z_{1} \equiv 2 H_{1}$ in each direction respectively. Here $H_{1}$ denotes the putative disk thickness at $r=R_{\text {in }}$. The disk models used here thus have a large aspect ratio compared to that expected in most astrophysical applications. For example in the case of protostellar disks one expects $H_{1} \sim 0.05 R_{\text {in }}$. However, computationally one can achieve higher resolution per scale height for thicker disks. In our case, for most of the computational domain, the resolution is then equivalent to or greater than the standard resolution often used in local disk simulations of MHD turbulence (e.g. Hawley et al. 1996; Papaloizou et al. 2005). Use of smaller $H_{1}$ and higher resolution in global simulations of the type considered here is prohibitive.

We consider 8 models. Models C, C1, C2, C3, B and E are fully three dimensional while models $\mathrm{C} 2 \mathrm{D}$ and $\mathrm{E} 2 \mathrm{D}$ are the two dimensional counterparts of models C and E. Models C, B and $\mathrm{E}$ are initiated with increasing magnitudes of kinetic energy in the radial direction corresponding to a linear normal mode of increasing amplitude. These models enable the effects of increasing nonlinearity to be studied. The latter two were initiated with the same magnitude of vertical kinetic energy. Model C1 was identical to model C apart from a lower initial magnitude of kinetic energy in the vertical direction. Model C2 was also identical to $\mathrm{C}$ apart from a longer wavelength dependence of the initial imposed vertical velocity. Model C3 was identical to $\mathrm{C} 2$ apart from a more extended outer radial boundary and a correspondingly extended distribution of radial kinetic energy. These models enable the connection to linear theory to be studied.

For our models the equation of state together with the initial density distribution imply that the pressure, $P$, is constant, accordingly the models are set in a state of Keplerian rotation with $\Omega=\sqrt{G M_{*} / r^{3}}$. As stated above for these models we adopted $h=c_{\mathrm{s}} \sqrt{r / G M_{*}}=0.12=$ constant.

We relate the results of the simulations of globally eccentric disks to the solution of the eigenvalue problem specified by the second order ordinary differential Eq. (12) that determines the normal modes described in Sect. 3.3.

From now on we adopt units of length and time to be $R_{\text {in }}$ and $\sqrt{R_{\text {in }}^{3} / G M_{*}}$ respectively. In these units, for our models the period of one retrograde rotation of the $m=1$ pattern associated with the fundamental mode appropriate to all models other than C3 is $P_{P}=2 \pi / \Omega_{P}=455.2$.

To initiate a globally eccentric disk we add in a multiple of the linear fundamental eigenfunction to the disk state variables which is chosen to give a required amount of kinetic energy
Table 1. This table gives initial parameters for the models considered. The first column gives the model identifier. The second column gives $E_{r z}=\left(\int \rho\left(v_{r}^{2}+v_{z}^{2}\right) \mathrm{d} V\right) /\left(\int \rho c_{\mathrm{s}}^{2} \mathrm{~d} V\right)$ being the ratio of the initial kinetic energy associated with motion in the radial and vertical directions to the kinetic energy that these motions would have were the resultant velocity sonic. The third column gives $E_{z}$ the corresponding quantity when only vertical motions are considered. The fourth fifth and sixth columns give the number of grid points in the radial, azimuthal and vertical directions respectively. Note that models C2D and E2D are two dimensional such that there is no dependence on $z$. Hence there is no entry in the sixth column for these.

\begin{tabular}{llllll}
\hline \hline Model & $E_{r z}$ & $E_{z}$ & $N_{r}$ & $N_{\phi}$ & $N_{z}$ \\
\hline $\mathrm{C}$ & 0.36 & $1.66 \times 10^{-4}$ & 350 & 400 & 35 \\
$\mathrm{C} 1$ & 0.36 & $1.69 \times 10^{-5}$ & 350 & 400 & 35 \\
$\mathrm{C} 2$ & 0.36 & $1.66 \times 10^{-4}$ & 350 & 400 & 35 \\
$\mathrm{C} 3$ & 0.35 & $1.12 \times 10^{-4}$ & 450 & 400 & 35 \\
$\mathrm{~B}$ & 0.52 & $6.69 \times 10^{-4}$ & 350 & 400 & 35 \\
$\mathrm{E}$ & 1.27 & $6.69 \times 10^{-4}$ & 350 & 400 & 35 \\
$\mathrm{C} 2 \mathrm{D}$ & 0.36 & - & 350 & 400 & - \\
$\mathrm{E} 2 \mathrm{D}$ & 1.27 & - & 350 & 400 & - \\
\hline
\end{tabular}

initially associated with radial and vertical motions (see Table 1). If it was necessary in the initialization procedure, the density was prevented from decreasing below a floor of $8.8 \times 10^{-4}$ times the initial maximum value.

The kinetic energy associated with motion in the radial and vertical directions $E_{r z}$ is initially and subsequently dominated by the radial component so that to a good approximation we find that the maximum disk eccentricity $e_{\max } \propto \sqrt{E_{r z}}$, with $e_{\max }=0.1$ corresponding to $E_{r z} \sim 0.22$. Thus for models C, $\mathrm{C} 1, \mathrm{C} 2 \mathrm{D}$, and $\mathrm{E}, e_{\max }=0.13,0.13,0.13$, and 0.24 respectively.

As a central aim is to study the susceptibility of the models to a parametric instability which causes the excitation of inertial modes which are associated with vertical motion we need to introduce a vertical dependence into the state variables. This is done after having introduced the $m=1$ mode by subsequently adding a vertical velocity for $2<r<3.5$ given by

$$
v_{z}=C_{z} v_{\varphi} \cos \left(2 \pi \frac{\left(z-z_{1}\right)}{\left(z_{2}-z_{1}\right)}\right) \sin \left(k_{0}(r-2)\right) .
$$

Here $k_{0}=2 \pi \sqrt{3} /\left(z_{1}-z_{2}\right)$ for all models apart from C2 and C3 for which $k_{0}=2 \pi / 3$ and $C_{z}$ is a normalization constant chosen to give an appropriate initial kinetic energy associated with motion in the vertical direction $E_{z}$, see Table 1 . We also remark that for models other than $\mathrm{C} 2$ and $\mathrm{C} 3$ this initial vertical velocity is harmonic in both the radial and vertical directions with a ratio of radial to vertical wavenumber of $\sqrt{3}$. This is the expected ratio for the mode most naturally excited by parametric instability (see above and Paper I). In the case of models C2 and $\mathrm{C} 3$, the initial vertical velocity had a half wavelengh in the domain $(2,3.5)$ which is about twenty times larger than that expected for parametric instability in order to check the independence of any unstable behaviour to the form of the initial state variables. 

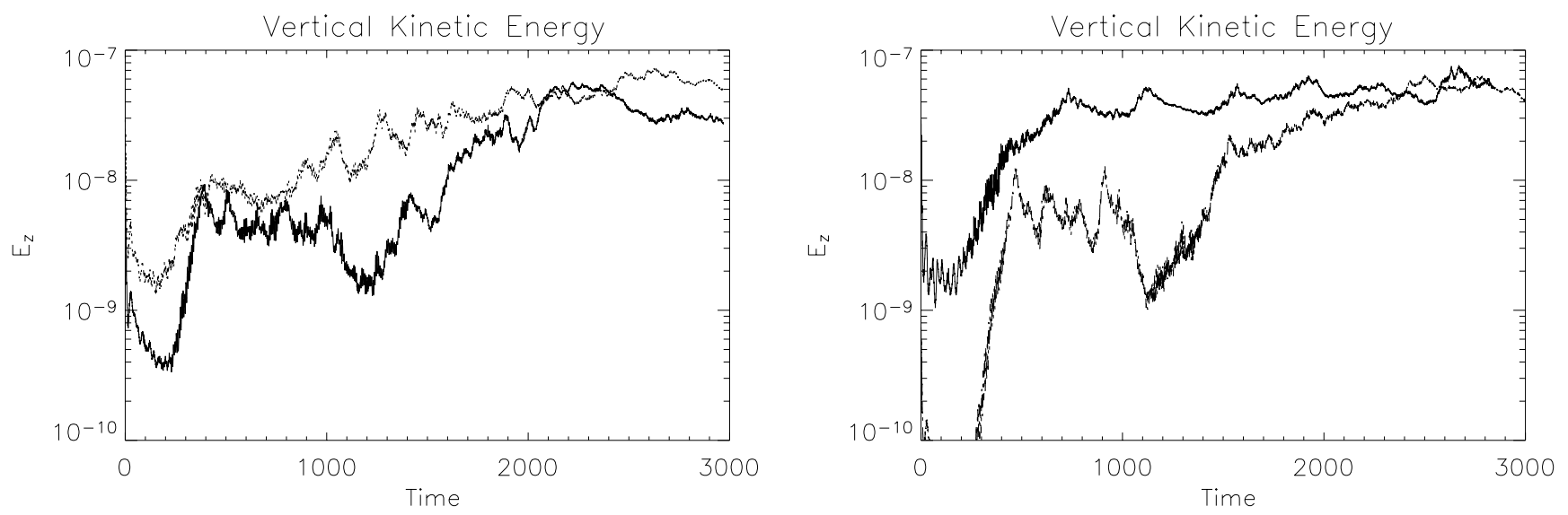

Fig. 1. This figure shows the vertical kinetic energy in arbitrary units as a function of time. The left panel is for models B (upper dotted curve) and $\mathrm{C}$. The right panel is for models $\mathrm{E}$ (initially upper and fuller curve) and $\mathrm{C} 1$. In these units the value of the kinetic energy of the initial fluid distribution if it were to move at the sound speed is $\int 0.5 \rho c_{\mathrm{s}}^{2} \mathrm{~d} V=4.38 \times 10^{-5}$. The final vertical kinetic energies reached correspond to a mean vertical velocity $0.03 c_{\mathrm{s}}$ throughout the disk.

\subsection{Numerical results}

After initiation all three dimensional models show an initial decrease of vertical kinetic energy, until parametric instability sets in and growth occurs. As time goes on the growth rate decreases and eventually the vertical kinetic energy levels off after about 2000 time units. In Fig. 1 we plot the vertical kinetic energy in arbitrary units as a function of time. for models B, C, E and C1. To normalize the magnitude of the kinetic energies involved we note that the value of the kinetic energy of the initial fluid distribution moving at the sound speed is $\int 0.5 \rho c_{\mathrm{s}}^{2} \mathrm{~d} V=4.38 \times 10^{-5}$. Thus the final vertical kinetic energies reached correspond to a local root mean square vertical velocity $\sqrt{\overline{v_{z}^{2}}} \sim 0.03 c_{\mathrm{s}}$ throughout the disk. In all cases and at all times the vertical kinetic energy is typically one thousandth of the radial kinetic energy.

\subsubsection{Growth of parametric instability}

In Paper I we showed that streamlines that are Keplerian ellipses are unstable to a parametric instability that is localized on streamlines. For small eccentricities and negligible pattern speed, $\Omega_{P}$, the instability can be regarded as the interaction of two inertial modes with angular frequencies, viewed from the frame corotating with the almost circular streamline, of $\pm \Omega / 2$.

The two modes must have azimuthal mode numbers $m$ differing by one in magnitude. The ratio of radial to vertical wavenumber is approximately $\sqrt{3}$. The longest possible vertical wavelength is controlled by the extent of the vertical domain and is $2 \pi / K_{z, \min }=2 H_{1}=0.24$. The radial wavelength corresponding to this is $0.24 / \sqrt{3}=0.14$. The vertical wavenumber $K_{z}$ must be a multiple of $K_{z, \min }$.

In Paper I the growth rate for small eccentricity, $e$ was found to be $\gamma=3 e \Omega / 16$. The application of the above results to the simulations is complicated by the presence of shocks induced by the presence of the inner boundary. Nevertheless a characteristic growth rate can be estimated by adopting $r=1.5$ which is where the radial velocity is a maximum. Adopting $e=0.13$ corresponding to model $\mathrm{C}$ we obtain $\gamma^{-1}=70$ time units corresponding to an energy growth time of 35 time units. A growth rate this fast was found only during the early phases of models $\mathrm{C}, \mathrm{C} 1, \mathrm{C} 2$, and $\mathrm{C} 3$, which started with the smallest ratio of non azimuthal kinetic energy to thermal energy, within the time interval 200-450 time units.

In order to investigate the effects of varying the profile of the initial conditions and the ratio of the inner to outer boundary radius we ran models $\mathrm{C} 2$ and $\mathrm{C} 3$. Model $\mathrm{C} 2$ is identical to model $\mathrm{C}$ but starts with a longer initial radial wavelength for the vertical velocity than model $\mathrm{C}$ which was tuned to the expected parametric instability. Model C3 has the same initial vertical velocity as model $\mathrm{C} 2$ but a radial domain extended by a factor 9/7. The time dependent evolution of the vertical kinetic energy for models C, C2 and C3 is plotted in Fig. 2. These attain similar growth rates of about 40 time units in the interval 200-450 time units, with models C2 and C3 doing so over a smaller time interval at later times. However, all these models evolve towards a similar state.

In Fig. 2 we also plot contours of $v_{z} /\left(\Omega H_{1}\right)$ in typical vertical sections for models $\mathrm{C}, \mathrm{C} 2$, and $\mathrm{C} 3$ at times $672.6,673.6$ and 404.5 respectively. These all show the develompent of structures in the inner regions with the expected vertical and radial wavelengths of 0.24 and 0.14 appropriate to the parametric instability. Although coherence is poor one can nonetheless see about seven radial wavelengths in the interval $(1,2)$ in the case of models $\mathrm{C}$ and $\mathrm{C} 2$. Such structures, although visible in model $\mathrm{C} 3$ are less extended because of the earlier time. In the case of model $\mathrm{C}$ which started with appropriately tuned initial conditions, the wavelengh of 0.14 can be seen throughout the radial domain. We comment that although in the above discussion we have focused on the behaviour of $v_{z}$, a very similar pattern of behaviour is found if one uses $v_{r}$ after subtracting out the vertically averaged value so that the dominant vertical wavelength is $2 H_{1}$.

However, for these and other models the growth slows and becomes erratic at later times. The slow down is likely to be because growth only occurs at larger radii then. The final 

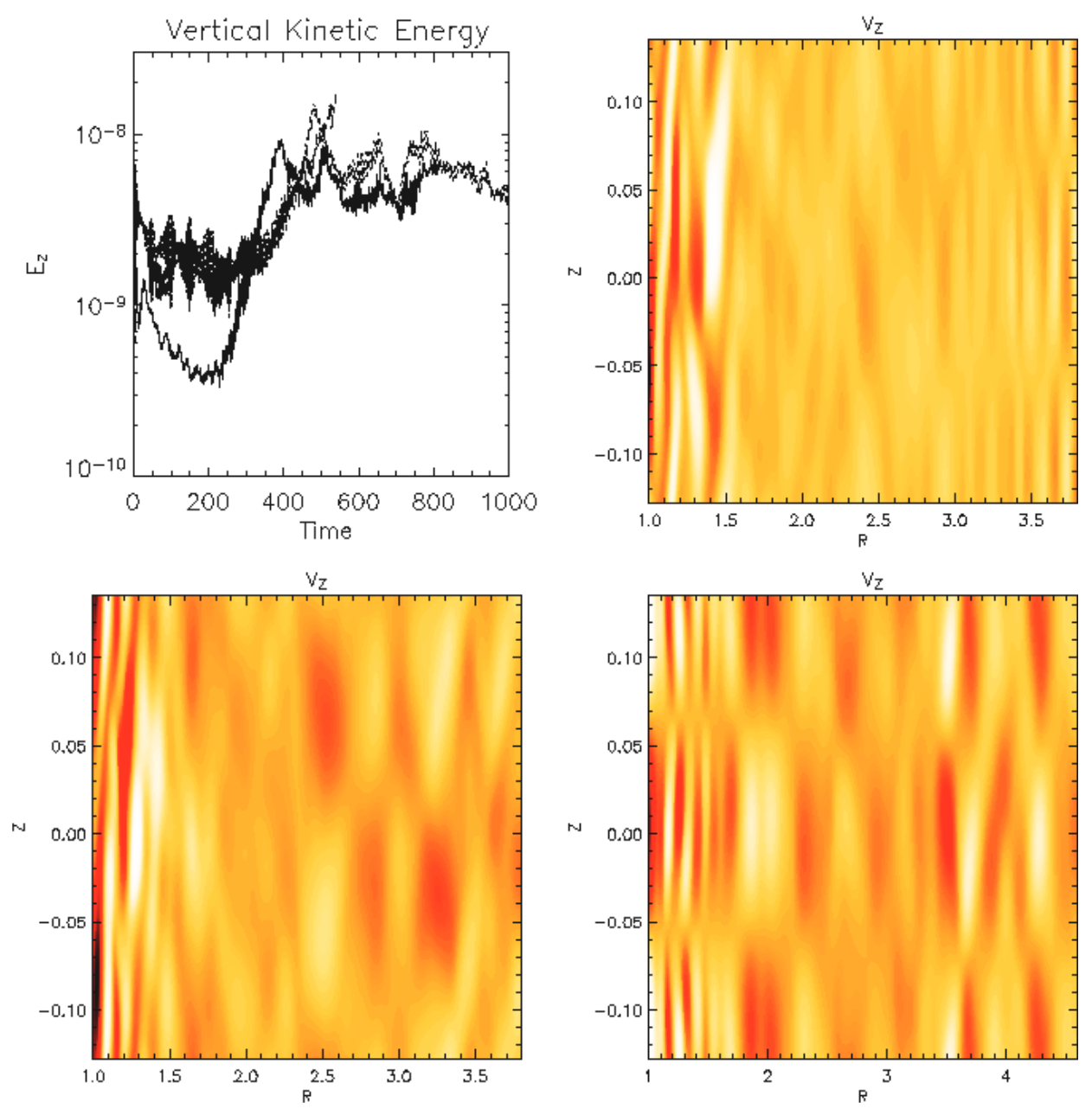

Fig. 2. The upper left panel shows the vertical kinetic energy in arbitrary units as a function of time for models $\mathrm{C}$ (initially lowest and full curve), C2 (dotted curve ) and C3 dashed curve. The initial distribution of radial kinetic energy in the inner regions of models C2 and C3 was very similar resulting in a similar evolution of vertical kinetic energy such that the corresponing curves intersect often. The upper right, lower left and lower right panels give contour plots of $v_{z} /\left(\Omega H_{1}\right)$ in typical $(r, z)$ sections for models $\mathrm{C}, \mathrm{C} 2$ and $\mathrm{C} 3$ at times $672.6,673.6$, and 404.5 respectively. A characteristic radial wavelength corresponding to the expected one of $2 H_{1} / \sqrt{3}$ is apparent at various locations (see text).

limiting value of the vertical kinetic energy corresponding to $\sqrt{\overline{v_{z}^{2}}} \sim 0.03 c_{\mathrm{s}}$ can be understood if we equate the characteristic instability growth rate to the eddy turn over rate $v_{z} /\left(2 H_{1}\right)$. Then $v_{z}=2 H_{1} \gamma \sim 3 e H_{1} \Omega / 8=3 e c_{\mathrm{s}} /(8 r)$. Using the maximum value for model C of $e=0.13$, we get $v_{z} \sim 0.05 c_{\mathrm{s}} / r$.

We illustrate the local scale of the turbulence in Fig. 3 in which contours of the vertical kinetic energy per unit mass, $0.5 v_{z}^{2}$, in the mid-plane are plotted for model $\mathrm{E}$ at the late time $t=2835$. The maximum vertical kinetic energy per unit mass shown is $8.67 \times 10^{-3}\left(0.5 c_{\mathrm{si}}^{2}\right)$ corresponding to $\left|v_{z}\right| \sim 0.1 c_{\mathrm{si}}$, with $c_{\mathrm{si}}$ being the sound speed at the inner boundary, and occurs near the inner boundary. The contours indicate elongation along elliptical curves as would be expected from the local stability theory of Paper I.

Figure 4 shows contours of the vertical kinetic energy per unit mass in a typical $(r, z)$ plane for model $\mathrm{E}$ at times $t=2140,2297,2333$, and 2835. These contours indicate turbulence ocuring on radial wavelengths similar to the value expected from local stability theory namely $2 H_{1} / \sqrt{3}$. On the other hand, there is evidence that somewhat larger scale structures can temporarily appear. This may be associated with nonlinear mode coupling.

In Fig. 5 we show the kinetic energy associated with motion in the $(r, z)$ plane as a function of time for three dimensional models which develop turbulence in comparison with their two dimensional counterparts for which vertical dependence of the sate variables was excluded. Although $E_{r z}$ decreases significantly, the amount of decrease is almost the same in the two and three dimensional cases indicating that effects that work without vertical dependence such as shocks are more important than the turbulence for inducing dissipation.

In the case of model $\mathrm{C}$ and its two dimensional counterpart $\mathrm{C} 2 \mathrm{D}, E_{r z}$ is $\sim 10$ percent less in the turbulent case after $\sim 3000$ time units while for model $\mathrm{E}$ and its two dimensional counterpart E2D the difference is 3 percent, the smaller indicating the greater importance of shocks in this case with initially larger $E_{r z}$.

We remark that Fig. 5 indicates the presence of time dependent oscillations in the radial kinetic energy. These can be traced to the presence of modes other than the fundamental mode with $m=1$. The short period oscillations belong to 


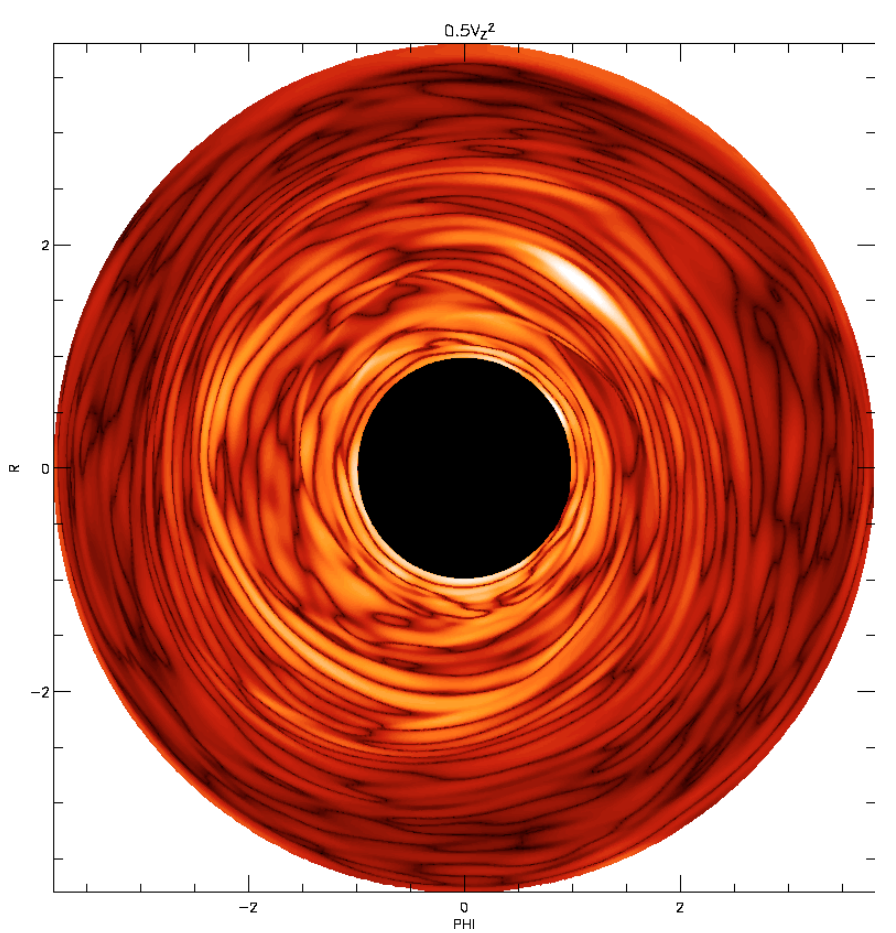

Fig. 3. This figure shows the vertical kinetic energy per unit mass $0.5 v_{z}^{2}$ found in the mid-plane for model $\mathrm{E}$ at time $t=2835$. Lighter shades correspond to larger values. The maximum vertical kinetic energy per unit mass in this plot is $8.67 \times 10^{-3}\left(0.5 c_{\mathrm{si}}^{2}\right)$. The smallest value is almost zero.

modes with higher $m$. In the case of models $\mathrm{C}$ and $\mathrm{C} 2 \mathrm{D}$ a long period of about 150 time units is discernable. This is consistent with being the beat period between the fundamental mode and the first overtone with $m=1$ (see Sect. 3.3). The existence of such a beat period is expected as a consequence of impure initial data. However, the overtone seems to be much more effectively damped in the higher amplitude models E and E2D.

\subsubsection{Shocks}

A feature of these simulations (both in 2D and 3D) is that the amount of kinetic energy initiated in the form of the linear $m=1$ mode is enough to induce significant shocks in the surface density profile that induce dissipation in all cases. The effect increases in importance as the magnitude of the initial radial kinetic energy increases. The shocks in these simulations are apparently induced by the rigid inner boundary. This acts as an obstacle to the inward finite amplitude radial motion associated with the disk eccentricity and shocks characteristic of a wake are produced. These are associated with some angular momentum transport and movement material towards the inner boundary. This is a natural result of circularization of the orbits of material close to the inner boundary.

These features are illustrated in Fig. 6 which shows plots of the azimuthally averaged surface density for model $\mathrm{C}$ for different times as the simulation progresses. A fairly stable form, with a modest peak at the inner boundary is attained after time 2000 units. Figure 7 gives the corresponding plot for the non turbulent two dimensional model C2D which displays similar behaviour.

The behaviour of model $\mathrm{B}$ which had larger initial radial kinetic energy amplitude is plotted in Fig. 8 and model E which had even larger initial amplitude is illustrated in Fig. 9. These show similar inner density features to model $\mathrm{C}$ but they become more pronounced for these initially higher amplitude and more active models.

Figure 10 shows the midplane density contours for model C at times $t=2119,2308,2609$, and 2968. The ratio of the maximum and minimum values of the density is 9.6. We comment that because of the lack of vertical stratification all horizontal planes always give very similar plots. Contour plots for the corresponding two dimensional model C2D are shown in Fig. 11 at times $t=1278,1379,1545$, and 2906. Midplane density contours for model B are plotted in Fig. 12 for times $t=$ 2075, 2227, 2610, and 2975 while Fig. 13 gives density contours for for model $\mathrm{E}$ at times $t=2140,2297,2333$, and 2835 respectively.

All of these plots indicate the presence of a global $m=1$ pattern that rotates slowly in the retrograde sense. They all also manifest the presence of trailing shocks emanating from the inner boundary as discussed above. We have estimated the rotation period to be $P_{P}=440$ time units in the case of models C and $\mathrm{C} 2 \mathrm{D}$ which is in good agreement with the linear eigenmode calculation which gave a period of $P_{P}=455.2$ time units. We have also detected a tendency of the determined period to shorten as the initial amplitude of the $m=1$ disturbance increases. Thus for models $\mathrm{B}$ and $\mathrm{E}$ we found $P_{P}=430$ and $P_{P}=400$ time units respectively. The pattern is observed to persist for the simulation run times over which there are $\sim 7$ full rotations.

We have also compared the vertically averaged $m=1$ Fourier component of the radial velocity with that obtained from the linear eigenmode calculation. The results are plotted in Fig. 14 for model C1, in Fig. 15 for model C2D, in Fig. 16 for model B and in Fig. 17 for model E.

As indicated by these plots, there is excellent agreement, particularly at early times between the simulation results and the linear eigenmode calculation for models $\mathrm{C} 1$ and $\mathrm{C} 2 \mathrm{D}$ which had both the smallest initial radial vertical kinetic energies. This agreement. although good, worsens for models B and E, which had larger initial radial and vertical kinetic energies, and for which the simulation eigenfunction was shifted to larger radii. Note also that the amount of matter close to the inner boundary is larger also. The latter situation is consistent with the decrease in pattern rotation period for those models.

\section{Discussion}

In this paper we have studied the evolution of global $m=1$ modes with low pattern speeds in a hydrodynamic model of a differentially rotating disk. The imposition of these modes on a disk for which the fluid orbits in circles corresponds to introducing an initial finite disk eccentricity. The modes initiated corresponded to a linear normal mode with amplitudes chosen to give maximum eccentricity in the range $0.13-0.24$. The associated radial velocities are typically sonic and shocks are 

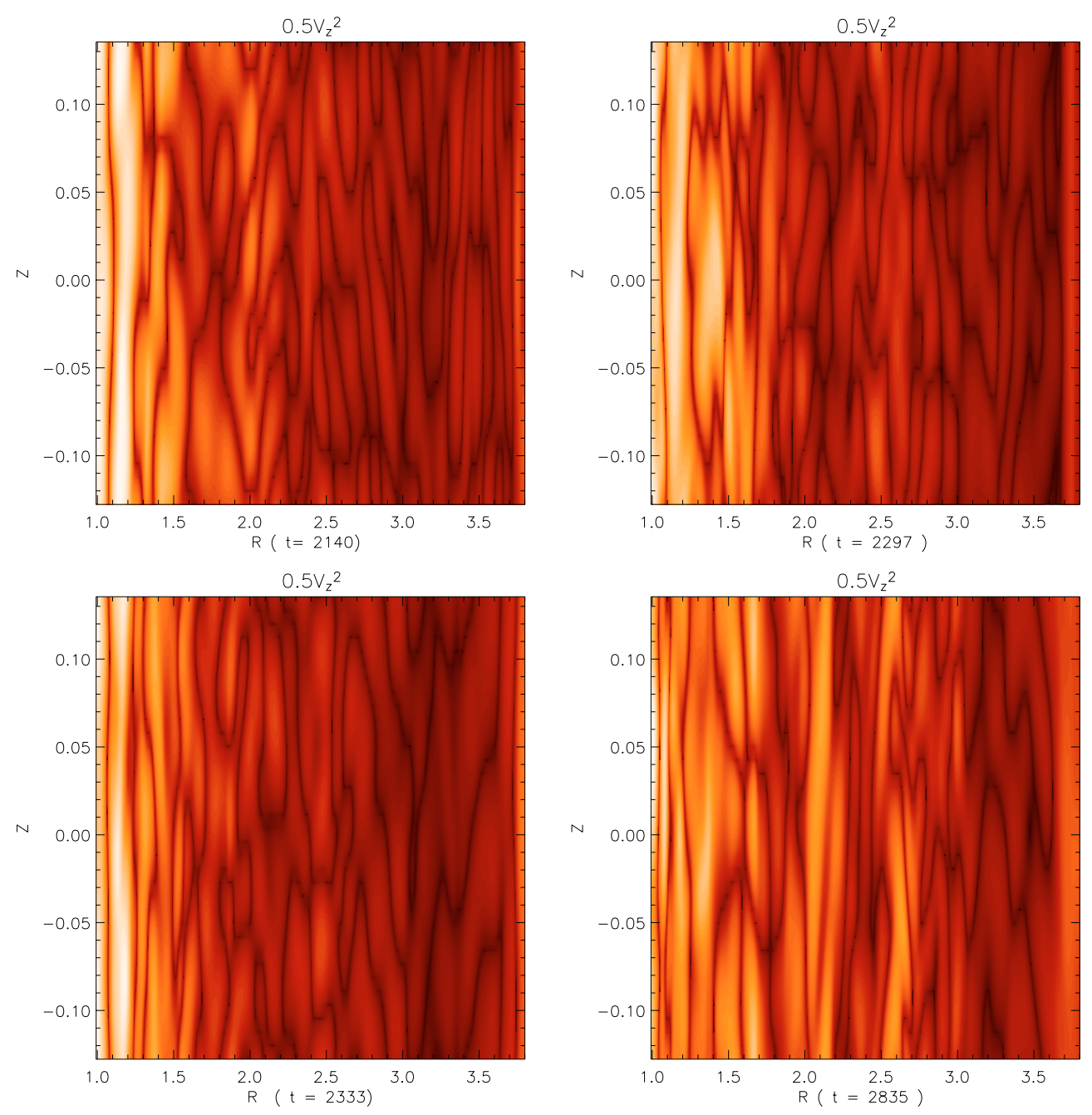

Fig. 4. This figure shows the vertical kinetic energy per unit mass $0.5 v_{z}^{2}$ found in a typical $(r, z)$ plane for model $\mathrm{E}$ at times $t=$ $2140,2297,2333$, and 2835. Lighter shades correspond to larger values. The maximum vertical kinetic energy per unit mass is $1.12 \times$ $10^{-2}\left(0.5 c_{\mathrm{si}}^{2}\right)$.
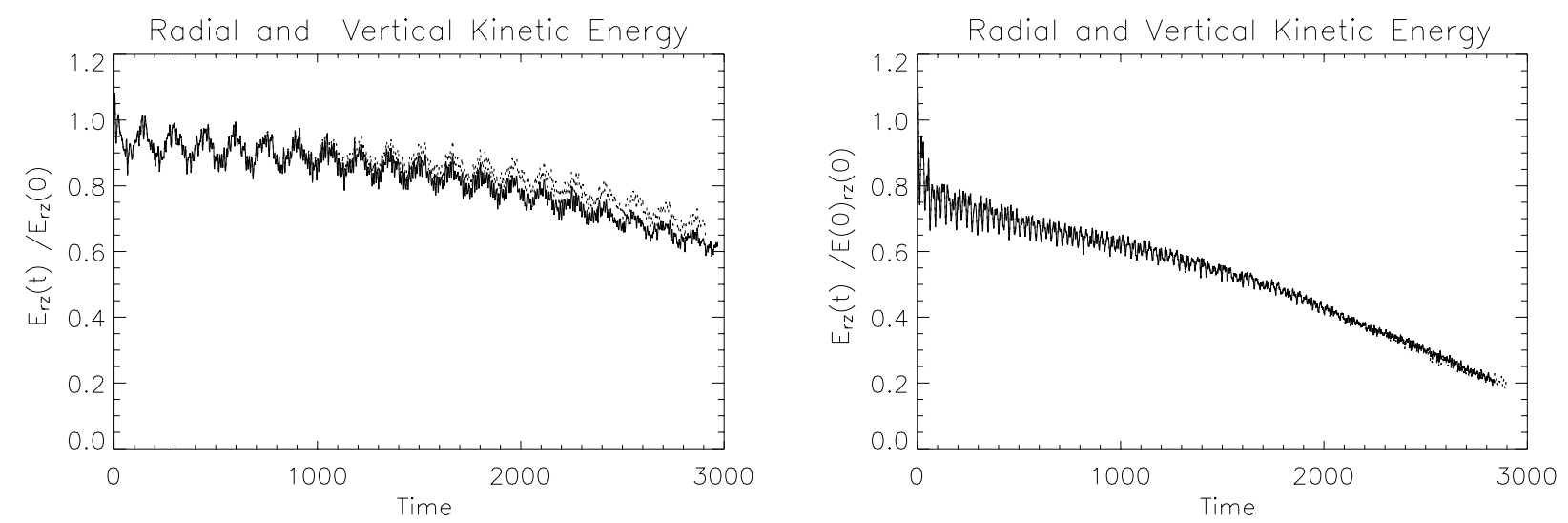

Fig. 5. This figure shows the kinetic energy associated with motion in $(r, z)$ as a function of time for three dimensional models with turbulence compared to their two dimensional counterparts for which vertical motion was suppressed. The left panel shows models C and C2D (dotted curve) while the right panel shows models E and E2D (dotted curve). The illustrated behaviour of the two and three dimensional models is very similar so that the kinetic energy plotted is barely distinguishable apart from at the very latest times where the three dimensional models have slightly smaller values. 


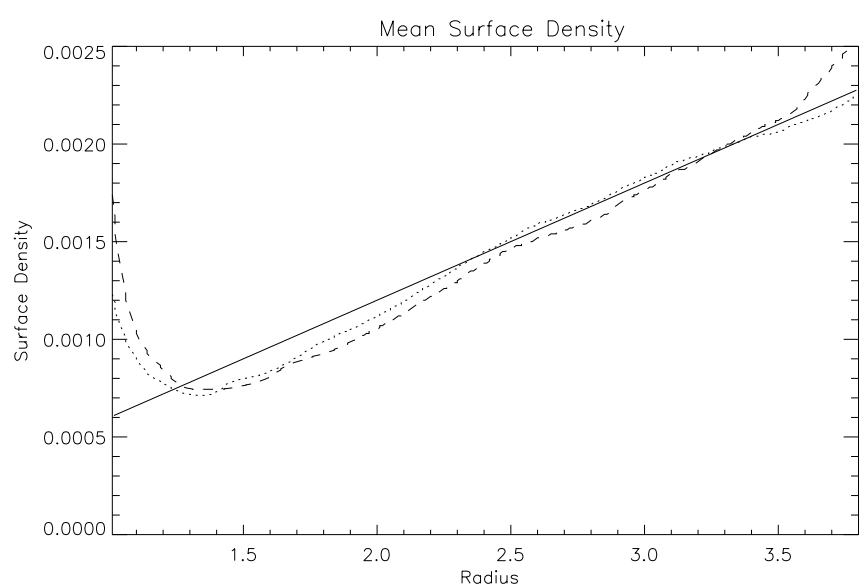

Fig. 6. This figure shows plots of the azimuthally averaged surface density for model C. These are for times $t=2119$, (dotted curve) and $t=2968$, (dashed curve). The full curve gives the initial surface density profile.

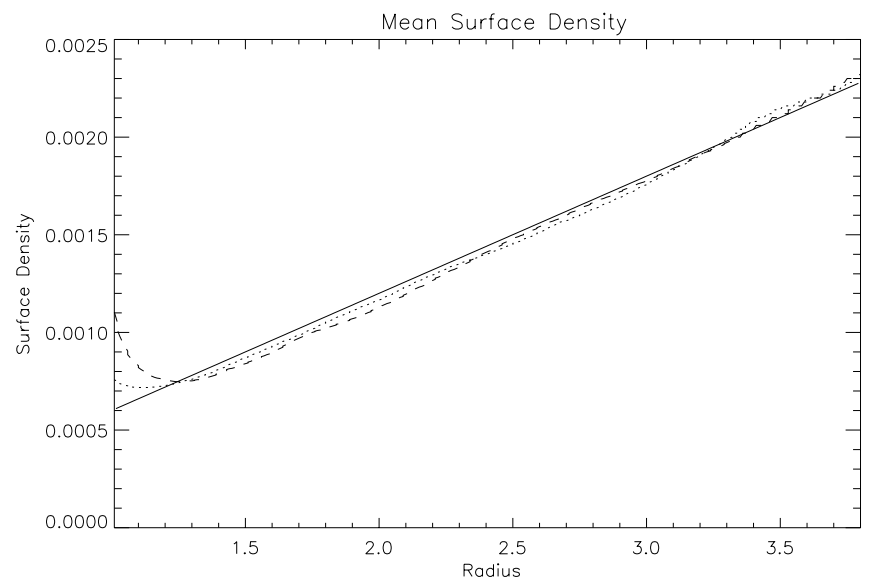

Fig. 7. As in Fig. 6 but for model C2D. The plots are for times $t=1278$, (dotted curve) and $t=2906$, (dashed curve).

induced near the inner radial rigid boundary which acts as an obstacle to the radial motion associated with eccentricity.

Nonetheless, it was found that the modes resulted in long lived patterns, lasting for the duration of the simulations, with slow retrograde precession period which was in good agreement with the predictions of linear normal mode analysis, the agreement being better for the lower amplitude smaller eccentricity models.

For three dimensional models it was found that there was the growth of a local instability to producing vertical motions that eventually resulted in low level turbulence with root mean square vertical velocity $\sqrt{\overline{v_{z}^{2}}} \sim 0.03 \mathrm{c}_{\mathrm{s}}$. However, the turbulence was not the major source of dissipation in the simulations presented here. Shocks induced at the inner boundary were more effective. Figure 5 shows these caused a reduction of mode energy of 40 percent for model $\mathrm{C}$ and an even greater 80 percent reduction for the higher amplitude model E. The presence of these shocks appears not to have affected the onset of parametric instability though their dissipation of energy most probably restricted the final amplitude of turbulence. In spite of the dominance of the shocks, Fig. 5, by providing

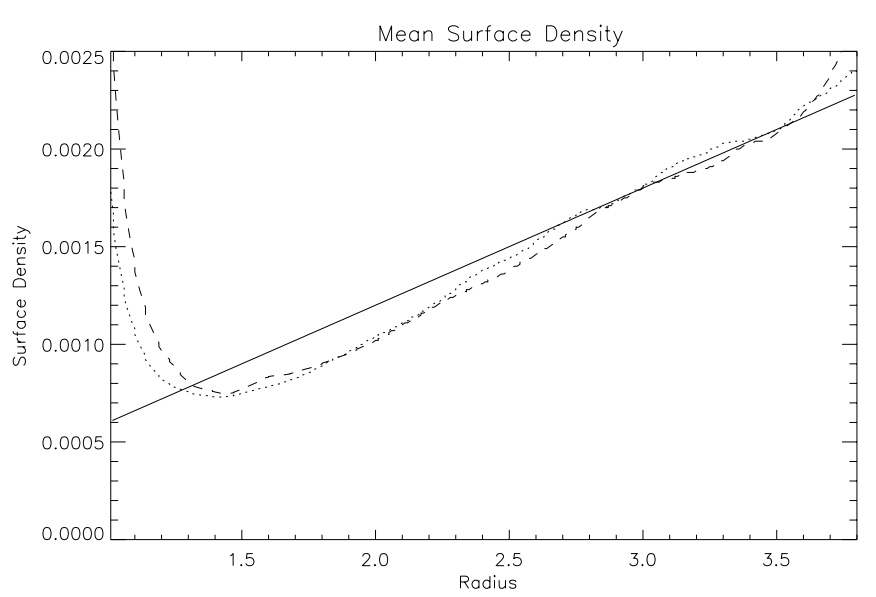

Fig. 8. As in Fig. 6 but for model B. The plots are for times $t=2075$, (dotted curve) and $t=2975$, (dashed curve). The full curve gives the initial surface density profile.

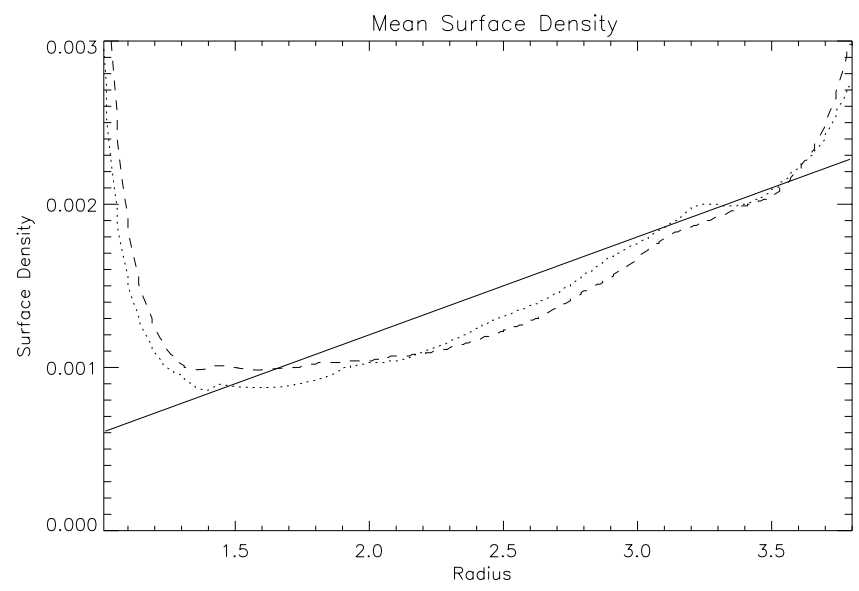

Fig. 9. As in Fig. 6 but but for model E. The plots are for times $t=2140$, (dotted curve) and $t=2835$, (dashed curve). The full curve gives the initial surface density profile.

a comparison of turbulent 3D models with non turbulent 2D models, provides some evidence of additional dissipation due to turbulence. We crudely estimate an additional 5 percent of the initial $m=1$ mode kinetic energy was dissipated in the model $\mathrm{C}$ simulation. This corresponds to an amplitude decay time of $t_{\mathrm{d}}=1.2 \times 10^{5}$ time units.

If we interpret this as resulting from an effective turbulent viscosity $v=\alpha h^{2} r^{2} \Omega$, acting globally over the disk such that $t_{\mathrm{d}}=r^{2} / v$, evaluating $\Omega$ at a mean radius $r=2$, we obtain an effective $\alpha \sim 10^{-3}$ that applies when the characteristic disk eccentricity $\sim 0.1$ and the maximum radial velocity associated with the free eccentricity is approximately sonic.

But it is important to note that this $\alpha$ should not be interpreted as a standard Shakura \& Sunyaev (1973) viscosity parameter as the turbulence is here driven by non circular streamlines rather than shear (we have verified that we see no turbulence develop for models with circular streamlines subject to a small perturbation imparting vertical motion).

The dynamical behaviour of disks with eccentricity is of interest in the context of extrasolar planets with high orbital 

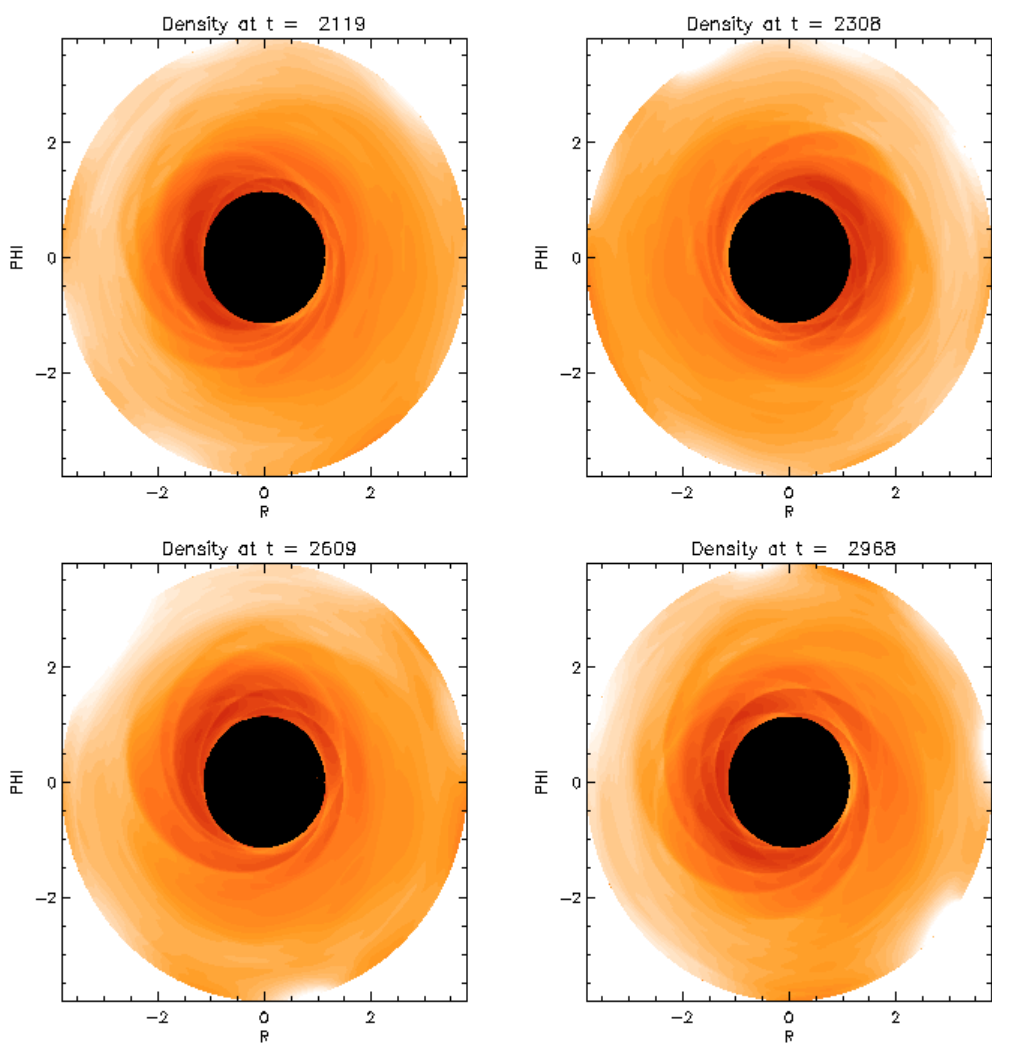

Fig. 10. The figure shows midplane density for model C at times $t=2119,2308,2609$, and 2968. Lighter shade corresponds to larger density. The ratio of the maximum and minimum values of the density is 9.6. Because of the lack of vertical stratification all horizontal planes give very similar plots.
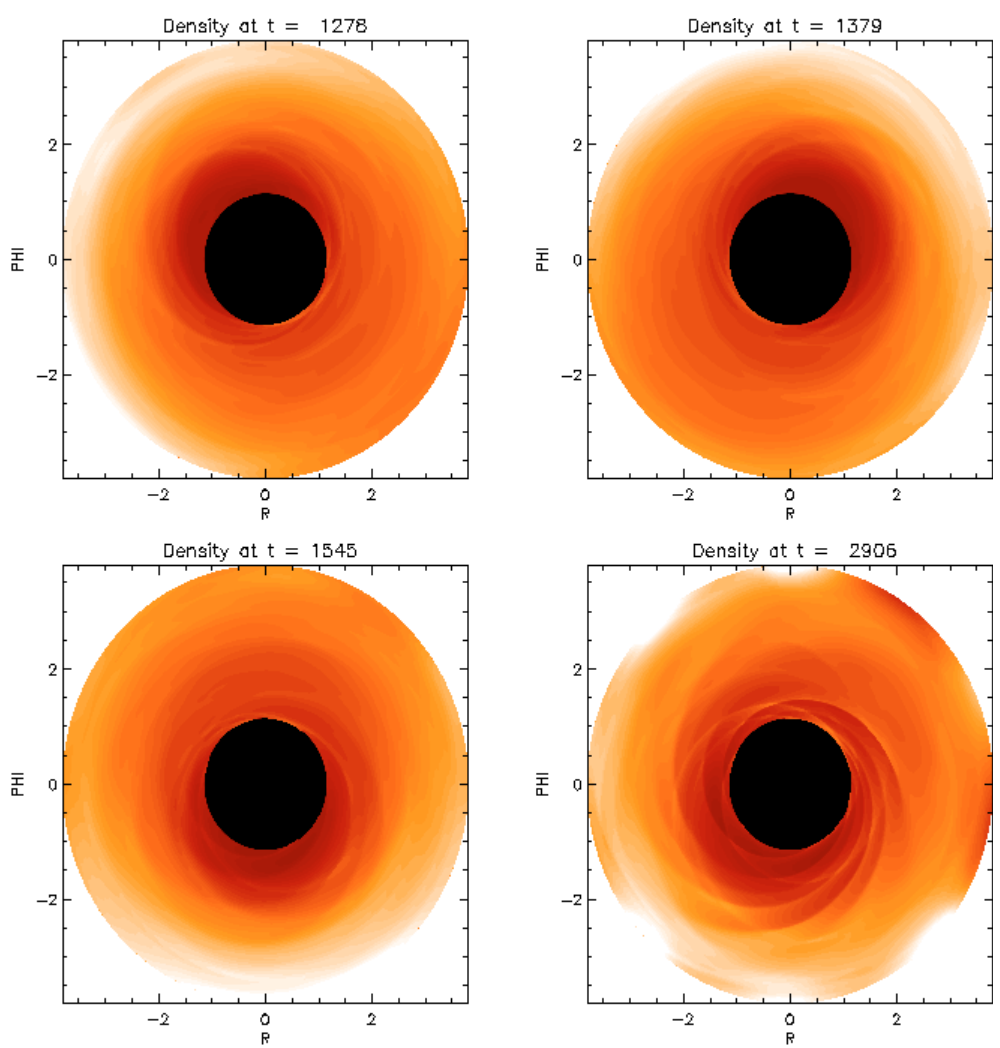

Fig. 11. As in Fig. 10 but for model C2D and for times $t=1278,1379,1545$, and 2906. 

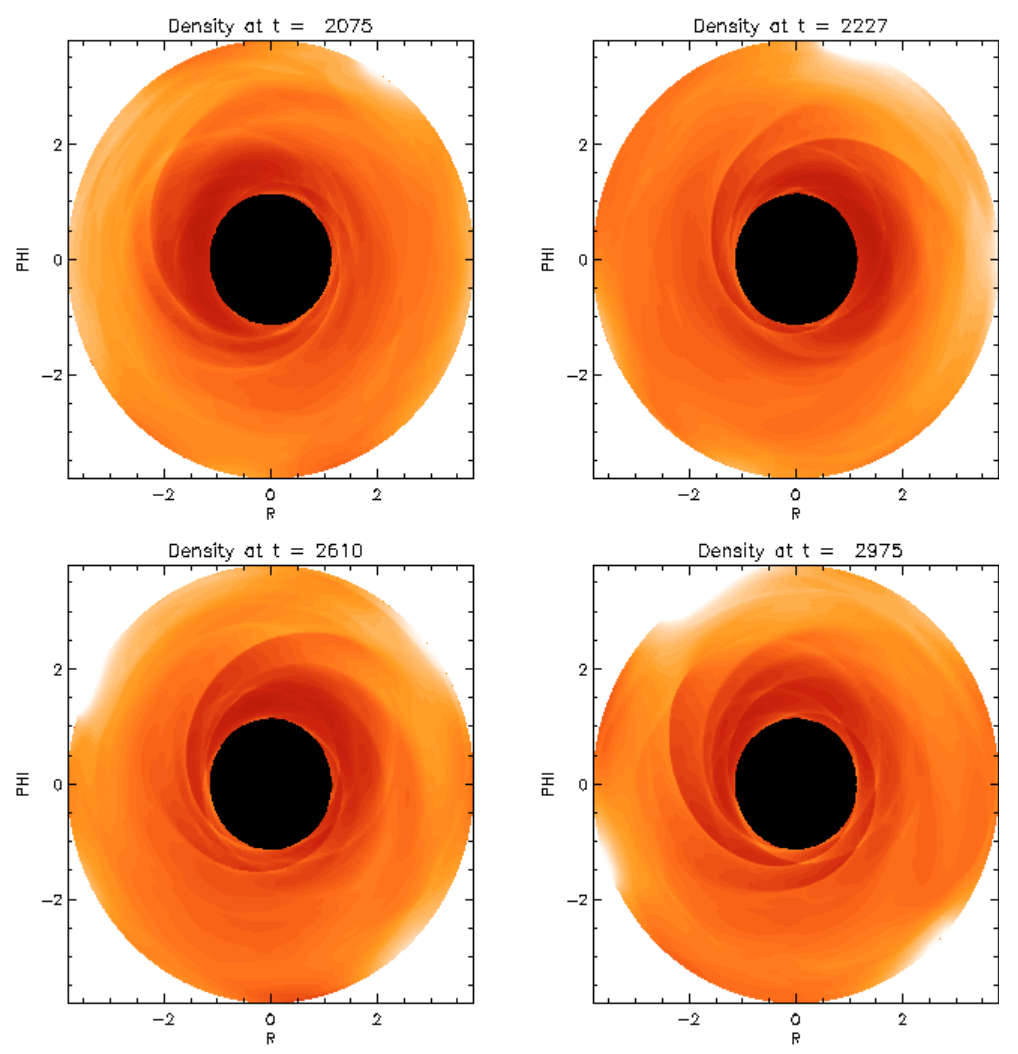

Fig. 12. As in Fig. 10 but for model B and for times $t=2075,2227,2610$, and 2975. The ratio of the maximum and minimum values of the density is 19.3 .
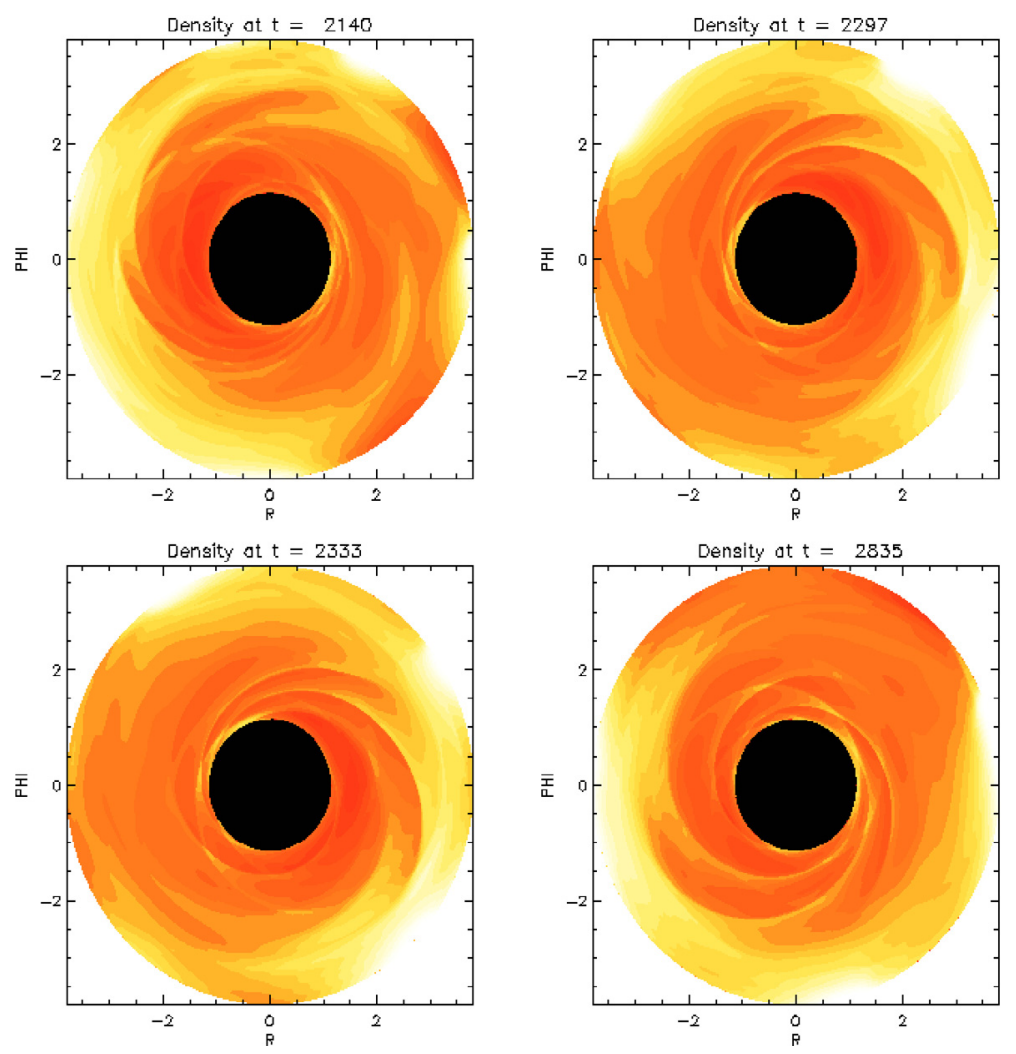

Fig. 13. As in Fig. 10 but for model $\mathrm{E}$ and for times $t=2140,2297,2333$, and 2835. The ratio of the maximum and minimum values of the density is 17.4 . 


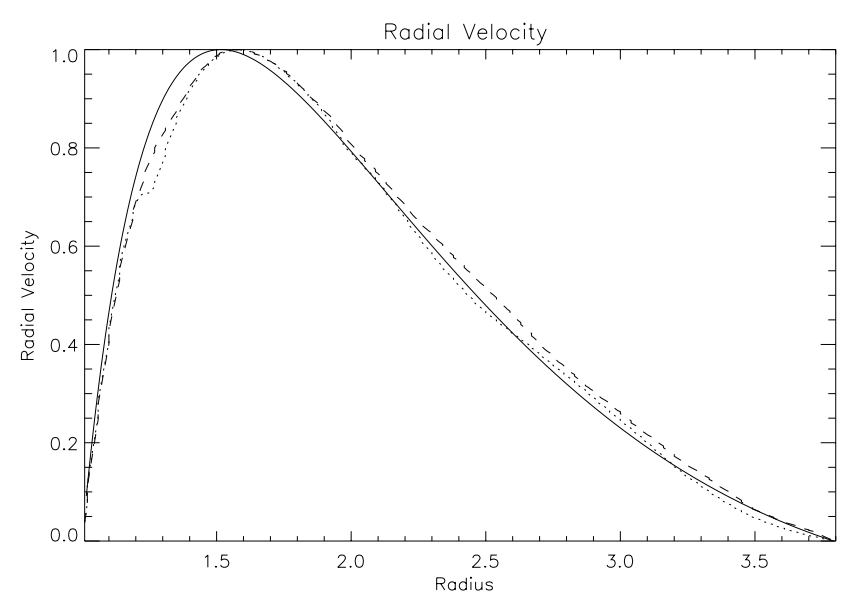

Fig. 14. This figure shows the vertically averaged amplitude of the $m=1$ Fourier component of the radial velocity normalized to a maximum of unity as a function of dimensionless radius for model $\mathrm{C} 1$. These are for times $t=140$, (dotted curve) and $t=598$, (dashed curve). The full curve gives the amplitude calculated directly from linear perturbation theory.

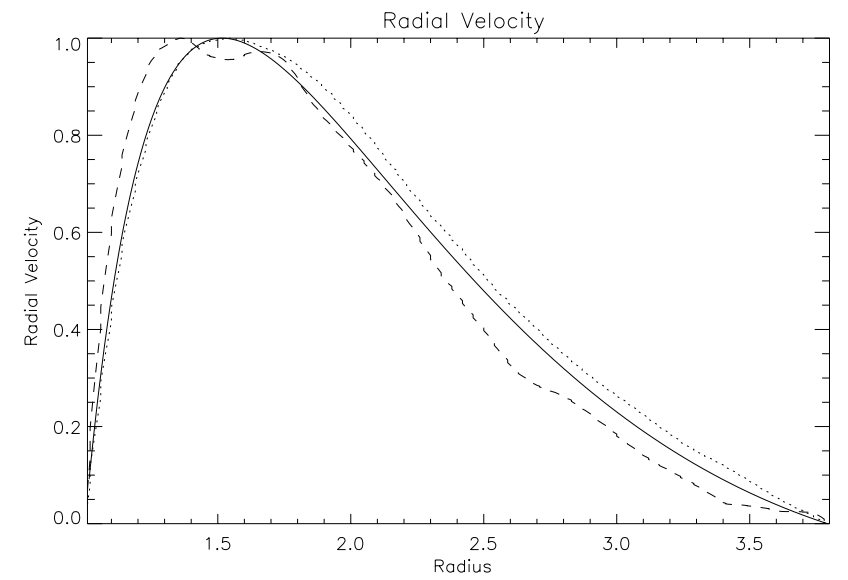

Fig. 15. As in Fig. 14 but for model C2D. The plots are for times $t=1278$, (dotted curve) and $t=2906$, (dashed curve). The full curve gives the corresponding function calculated directly from linear perturbation theory.

eccentricity (Mayor \& Queloz 1995; Marcy \& Butler 1998, 2000). This is because if the protoplanetary disk was present when the orbital eccentricities were attained, it would in general be expected to have some induced eccentricity as well. Turbulence induced through the natural instability of eccentric streamlines becomes relevant, especially in regions where magnetic fields are not important.

Although more realistic disk models will ultimately need to be considered, these results obtained here have potential implications with regard to the proposal of disk planet interactions as the origin of the excitation of the eccentricities of extrasolar planets (e.g. Goldreich \& sari 2003). In such a case the excitation of disk modes occurs naturally with the eccentricity of the planet orbit (e.g. Papaloizou 2002).

However, any excitation mechanism through for example Lindblad resonances (e.g. Lin \& papaloizou 1993) must overcome the dissipative processes discussed in this paper which apply even for non magnetic disks.

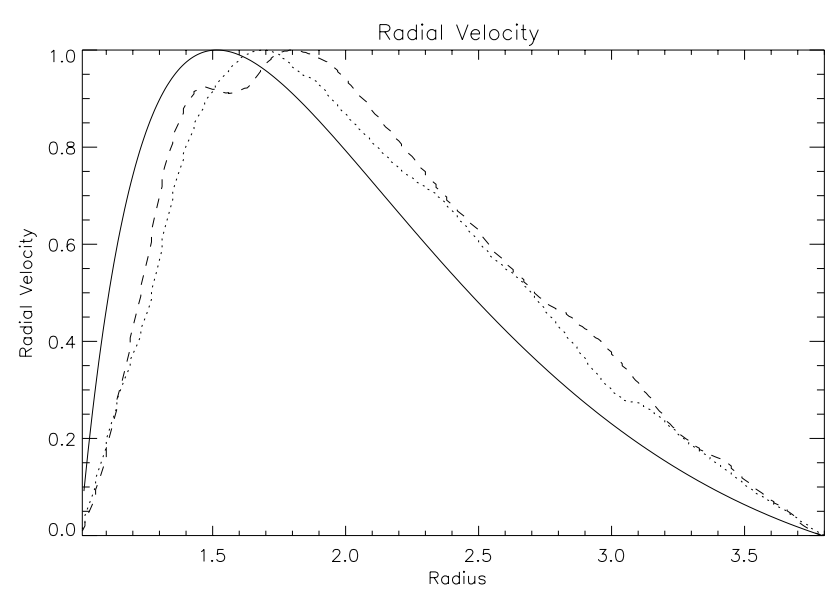

Fig. 16. As in Fig. 14 but for model B. The plots are for times $t=2075$, (dotted curve) and $t=2975$, (dashed curve). The full curve gives the corresponding function calculated directly from linear perturbation theory.

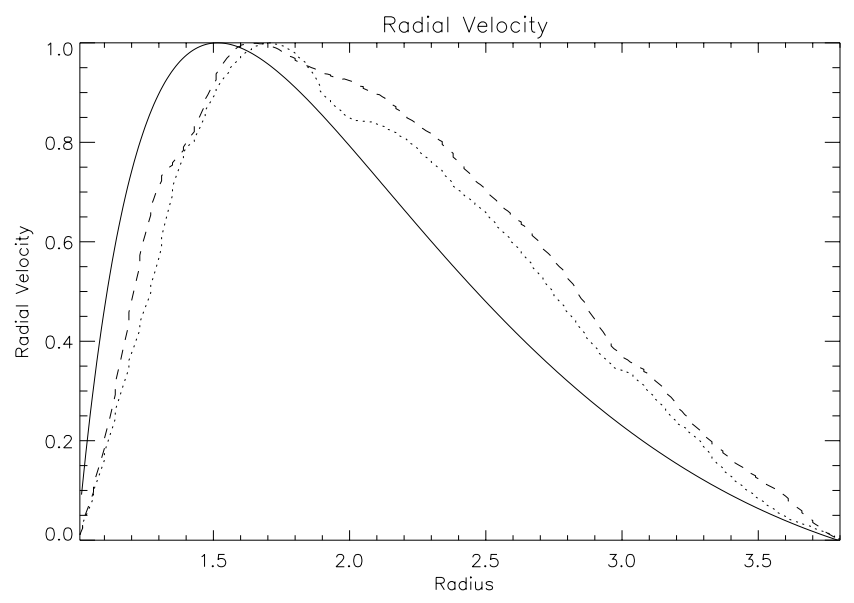

Fig. 17. As in Fig. 14 but for model E. The plots are for times $t=2140$, (dotted curve) and $t=2835$, (dashed curve). The full curve gives the corresponding function calculated directly from linear perturbation theory.

We first remark that for the mode amplitudes considered, turbulence induced by parametric instability does not lead to fast destruction of the disk eccentricity but rather a slow decay on a "viscous" timescale roughly estimated to correspond to an effective $\alpha \sim 10^{-3}$. This leads to an estimated timescale for the decay of eccentricity for a standard protoplanetary disk model with a central solar mass with $h=0.05$ at 5 AU of $10^{5-6} \mathrm{y}$. Accordingly this turbulence may be significant for evolutionary phenomena including the excitation of the eccentricity should they occur on such a timescale or longer.

However, we found that shocks were about an order of magnitude more effective at producing eccentricity decay for disks with typically sonic radial velocities. Although in our simulations these were clearly related to the adoption an inner rigid radial boundary, this may give similar restrictions on radial motion as the inner edge of a tidal gap. Note too that the decay of eccentricity may be even faster if magnetic fields are present and producing turbulence through the Magnetorotational instability (Balbus \& Hawley 1991). Accordingly the problem of 
eccentricity evolution in disk planet interactions requires consideration of a number of competing physical processes with the aid of 3D numerical simulations.

\section{References}

Artymowicz, P. 1992, PASP, 104, 769

Balbus, S. A., \& Hawley, J. F. 1991, ApJ, 376, 214

Bryden, G., Chen, X., Lin, D. N. C., Nelson, R. P., \& Papaloizou, J. C. B. 1999, ApJ, 514, 344

Goldreich, P., \& Sari, R. 2003, ApJ, 585, 1024

Hawley, J. F., \& Stone, J. M. 1995, Computer Physics Communications, 89, 127

Hawley, J. F., Gammie, C. F., \& Balbus, S. A. 1996, ApJ, 464, 690

Hawley, J. F. 2000, ApJ, 528, 462

Kley, W. 1999, MNRAS, 303, 696

Lin, C. C., \& Shu, F. H. 1969, ApJ, 140, 646

Lin, D. N. C., \& Papaloizou, J. C. B. 1993, Protostars and Planets III, 749 (Tucson, AZ: University of Arizona Press)
Nelson, R. P., Papaloizou, J. C. B., Masset, F., \& Kley, W. 2000, MNRAS, 318, 18

Nelson, R. P., \& Papaloizou, J. C. B. 2003, MNRAS, 339, 993

Nelson, R. P., \& Papaloizou, J. C. B. 2004, MNRAS, 350, 849

Marcy, G. W., \& Butler, R. P. 1998, ARA\&A, 36, 57

Marcy, G. W., \& Butler, R. P. 2000, PASP, 112, 137

Masset, F. S. 2001, ApJ, 558, 453

Mayor, M., \& Queloz, D. 1995, Nature, 378, 355

Ogilvie, G. I. 2001, MNRAS, 325, 2310

Papaloizou, J. C. B. 2002, A\&A, 388, 615

Papaloizou, J. C. B. 2005, A\&A, 432, 743

Papaloizou, J. C. B., \& Nelson, R. P. 2003, MNRAS, 339, 983

Papaloizou, J. C. B., Nelson, R. P., \& Masset, F. S. 2001, A\&A, 366, 263

Papaloizou, J. C. B., Nelson, R. P., \& Snellgrove, M. D. 2004, MNRAS, 350, 829

Steinacker, A., \& Papaloizou, J. C. B. 2002, ApJ, 571, 413

Shakura, N. I., \& Sunyaev, R. A. 1973, A\&A, 24, 337

Winters, W. F., Balbus, S. A., \& Hawley, J. F. 2003, ApJ, 589, 543

Ziegler, U., \& Rüdiger, G. 2000, A\&A, 356, 1141 